\title{
Hydrometra Simulation for VR-Based Hysteroscopy Training
}

\author{
R. Sierra ${ }^{1}$, J. Zátonyi ${ }^{1}$, M. Bajka ${ }^{2}$, G. Székely ${ }^{1}$, and M. Harders ${ }^{1}$ \\ 1 Computer Vision Laboratory, ETH Zürich, Switzerland \\ \{rsierra, zatonyi, szekely, mharders\}@vision.ee.ethz.ch \\ 2 Clinic of Gynecology, Dept. OB/GYN, \\ University Hospital of Zurich, Switzerland
}

\begin{abstract}
During hysteroscopy a hydrometra is maintained, i.e. the uterus is distended with liquid media to access and visualize the uterine cavity. The pressure and flow induced by the liquid are crucial tools for the gynecologists during surgery to obtain a clear view of the operation site. This paper presents two different aspects of hydrometra simulation, namely the distension of the uterine muscle and the liquid flow simulation in the cavity. The deformation of the organ's shape is computed offline based on finite element calculations whereas the flow is approximated on the fly by solving the simplified Navier-Stokes equations. The real-time capabilities of the presented algorithms as well as the level of fidelity achieved by the proposed methods are discussed.
\end{abstract}

\section{Introduction}

Hysteroscopy, the endoscopic inspection of the uterus, has become an established technique in gynecological practice [2]. A fundamental prerequisite of the method is the proper distension of the uterine cavity, also known as hydrometra. In general, a non-ionic solution will be used for therapeutic procedures. In- and outflow of the distension fluid is accomplished via the endoscopic tool and controlled with valves, while the pressure of the liquid is provided by a pump. It is essential to select correct pressure settings for the hydrometra according to muscle tone and uterine wall thickness [9]. Additional to the inflation of the uterus, the fluid flow also ensures a clear visibility in the cavity during interventions. Obscurations can be caused by endometrial bleeding, floating tissue fragments or air bubbles. It is the gynecologist's experience to correctly dose the in- and outflow through the valves that leads to a proper view of the scene. According to [7, a number of complications can be encountered related to the application of the distension fluid. Thus, the total amount of liquid loss should be strictly limited to $1500 \mathrm{ml}$, at which point the procedure has to be terminated.

Due to these reasons, the correct handling of the valves is a crucial skill that has to be an integral part of training for every gynecologist. Virtual Reality based surgical simulation offers a promising alternative to today's teaching approaches, which are largely based on interventions performed on real patients. In order to provide a suitable learning environment, the degree of realism necessary for 
effective training of complete surgical procedures with a simulator has to be identified. Our current research aims at the development of a simulator of highest possible realism for the procedural training of hysteroscopy. Key elements of this system are the proper cavity inflation, flow control of the distension fluid, pressure adjustment to handle small bleeding, and correct management of liquid pressure.

\section{Related Work}

In this paper an integrated approach for real-time simulation of various aspects of generating and maintaining of the hydrometra is presented. The authors are not aware of work with a similarly comprehensive approach. Nevertheless, related research exists, which focuses on individual aspects of our method.

A number of different approaches has been suggested for the description of the mechanical behavior of organic tissue. Usually, for these methods a trade-off has to be found between accuracy and computation time. Free-form deformation (FFD) techniques, stemming from Computer Graphics [11, have been applied in surgical simulation due to their efficiency. However, FFD procedures have only very limited physical meaning. More accurate deformations can be obtained with mass-spring models, initially introduced in [15. Numerous projects have made use of this method, however, problems remain due to its limitations when approximating true physics. Nevertheless, an advantage of the method is its applicability in real-time simulations. Finally, rigorous representation of soft tissue physics can be achieved with continuum mechanics approaches, the most popular one being the Finite Element Method (FEM). Different levels of accuracy for deformation simulation have been realized with FEM, ranging from elastic linear [4] to nonlinear anisotropic systems [10]. Recently, a simulation of a hydrometra using FEM has been presented which, however, requires several hours of computation [17.

Physically-based real-time simulation of fluid flow in 3D has found increasing attention in recent years, mainly fueled by ever increasing computing power. In [3], discretized Navier-Stokes equations are solved using explicit finite differences and visualized with a so-called marker particle approach. The method requires relatively small time steps to ensure stable simulation, thus limiting its usage in real-time applications. A solver based on a semi-Lagrangian advection scheme has been presented in [14. Its unconditional stability allows using larger time-steps, hence making fluid simulation in real-time possible. Recent work aims at efficient GPU-based implementations of fluid solvers to increase computational speed [6]. We previously reported on a method that combines the semi-Lagrangian solver with the marker particle approach to represent the blood flow in a simple geometry [18. This combination allowed to achieve more realistic liquid-like behavior. However, the method was tailored to only visualize blood streams in a static environment. The results are extended in the following to consider the complex dynamic scene when regulating pressure and flow conditions during hydrometra. 


\section{Real-Time Deformation of the Uterine Cavity}

The almost instantaneous response of the uterine cavity to pressure adjustments of the distension fluid exceeds by far the real-time capabilities of most known deformation models. Not only computationally intensive approaches, like FEM, are therefore completely infeasible, but even mass-spring methods proved intractable for the given problem. Firstly, the deformation accuracy of the latter is limited, and suitable model parameters can not easily be found. Secondly, in order to precisely approximate the shape of the uterus, still a large number of mesh elements is needed. As a result, the required mesh updates are currently beyond the capabilities of mass-spring methods. Therefore, free-form deformations seem to be better suited to solve the problem at hand. However, they do not provide the needed physical realism.

In order to achieve physically realistic real-time deformation of the uterine cavity, we suggest the combination of accurate FEM computations with FFD approaches. The underlying idea is to first carry out offline precomputations to obtain an accurate response of the tissue model to the fluid pressure. These data are then used during real-time interaction for model adjustments based on given pressure states. It is assumed that the boundary conditions of the deformation model do not change during the intervention. This is usually the case in hysteroscopic procedures, since the surrounding tissues are not directly accessible. Moreover, extreme modifications of the cavity wall should be avoided. This is also fulfilled, since cutting into the myometrium is limited to a relatively thin layer during surgery, otherwise risking the perforation of the wall leading to the immediate termination of the intervention. Hence we can assume, that the uterus' response to pressure differences stays relatively constant throughout the intervention.

Three major steps are needed for our approach: Data acquisition from real organs for model geometries and material laws, offline computations with a highly accurate FE approach, and real-time replay of these data during the intervention. The first step consists of a statistical model which encodes the natural variability of the healthy organ geometry which was segmented from MRI data obtained from a study with volunteers 13 . Based on a predefined set of parameters that are familiar to the gynecologists, new surface meshes of the uterus can be derived intuitively prior to training. Material parameters for the offline deformation computations are obtained in-vivo with a tissue aspiration device 8]. The obtained data are fed into the FE model in order to achieve a reasonable approximation of real deformation behavior. Both the FEM computation and the real-time simulation are based on homogeneous tetrahedral meshes. While in the former stage the mesh is required for calculating the deformations, the tetrahedral representation is needed for collision detection and haptic feedback in the latter step. The specific mesh generation approach employed is presented in 12 . In the second step, the offline computation enables the use of more advanced, and thus more accurate, tissue modeling techniques. We apply the FEM presented in [17, where homogeneous, isotropic and nonlinear hyper-elastic material laws are used. Based on the in-vivo aspiration experiment a polynomial strain-energy function of the form 


$$
\Psi\left(\overline{I_{1}}, J\right)=\sum_{p=1}^{N} \mu_{p}\left(\overline{I_{1}}-3\right)^{p}+\frac{1}{2} \kappa(J-1)^{2}
$$

is used to describe the mechanical behavior of the particular soft tissue where $\bar{I}_{1}$ is the first reduced invariant of the right Cauchy-Green tensor, $J$ the volume ratio, $\mu_{p}$ material parameters and $\kappa$ the bulk modulus. $J \approx 1$, i.e. the material is assumed to be quasi-incompressible. The resulting equations are solved with the commercial package MarcMentat ${ }^{\mathrm{TM}}$. The organ model deformations are obtained for 100 different pressure settings. In the third step, the precomputed deformation states are loaded into the simulator and interpolated in real-time according to the applied fluid pressures. As the mesh topology does not change during deformation, only the vertex positions $\mathbf{x}$ of the tetrahedral mesh have to be updated based on the interpolation parameter $t$ and a limited number $n$ of precomputed instances $\mathbf{x}_{i}: \mathbf{x}=f\left(t, \mathbf{x}_{0}, \ldots, \mathbf{x}_{n}\right)$. Therefore, large meshes consisting of more than 30'000 tetrahedra and 50 interpolation samples can be updated in real-time. Already simple linear interpolation with as few as four precomputed samples proved to be sufficient for obtaining convincing visualizations.

\section{Handling of Distension Fluid}

The motion of liquid is described by the Navier-Stokes equations. For an incompressible fluid they take the form:

$$
\begin{aligned}
\nabla \cdot \mathbf{v}_{f} & =0 \quad \text { and } \\
\frac{\partial \mathbf{v}_{f}}{\partial t} & =-\left(\mathbf{v}_{f} \cdot \nabla\right) \mathbf{v}_{f}+\nu \nabla^{2} \mathbf{v}_{f}-\frac{1}{\rho} \nabla p+\mathbf{F}
\end{aligned}
$$

where $\mathbf{v}_{f}$ is the velocity vector, $p$ is the pressure, $\rho$ is the density of the fluid, $\nu$ represents the kinematic viscosity coefficient and $\mathbf{F}$ corresponds to the vector of external body forces. The density and the temperature of the fluid are considered constant. Equation (11) represents the mass conservation in the fluid, while (2) is the momentum equation [1]. Equations (1) and (2) are solved numerically on a uniform Cartesian grid to evaluate the time dependent behavior of the fluid.

In order to solve the velocity field, proper boundary conditions have to be set. For hysteroscopy these are the endometrium as well as pathologies present in the scene, the in- and outflow according to the tool position, and their intensity according to the valve states. By labeling the voxels of the grid, these elements can be incorporated consistently into the numerical solver as discussed in [3]. As both the hydrometra and the pathologies may frequently change during simulation, the labeling of the voxels has to be updated accordingly. Therefore all voxels are re-labeled whenever the surfaces are modified in real-time [12. For the inflow, constant velocity is set at the voxels corresponding to the tip of the tool. The outflow is represented by voxels that are set to velocities according to the outflow conditions at the respective positions.

Depending on the state of the valves, the properties of the liquid current vary in a wide range. The maximal flow speed of the distension liquid at fully opened 
valves reaches approximately $2 \frac{\mathrm{m}}{\mathrm{s}}$ according to our measurements. Considering the small size of a normal cavity in the order of a few $\mathrm{cm}^{3}$, the liquid flow is highly turbulent with a Reynolds number of about 30'000. However, the direct numerical simulation of turbulent flows is still a challenging task and physically based simulation approaches require extremely time-consuming calculations. In order to improve the simulation fidelity, the swirling motion within the fluid caused by the turbulence has still to be modeled. Therefore the velocity field $\mathbf{v}_{f}$ serves as a realistic approximation for moderate flow speed. For higher flow rates, artificial vortices can be added as needed. While this combination is not physically accurate, it provides a higher level of fidelity in real-time.

At the start of the simulation, we place the initial position of the vortices randomly within the fluid filled area. The orientation $\omega$ of the vortices is given by the curl of the velocity field $\mathbf{v}_{f}$ interpolated at the position of the vortex: $\omega=\nabla \times \mathbf{v}_{f}$. The Rankine vortex model is used, where a concentrated vorticity is simulated in the core region and combined with an exponential decay of the circumferential velocity as the distance from the core increases [5]. The velocity field represented by the Rankine vortex is divergent free, i.e. satisfies (1), and therefore provides a kinematically plausible velocity field. During simulation, the vortices are moved in each iteration according to their vorticity vector $\omega$ by a simple Euler step, i.e. $\mathbf{p}_{\text {vort }}{ }^{t+1}=\mathbf{p}_{\text {vort }}{ }^{t}+\Delta t_{\text {vort }} \omega$. The resulting flow velocity can finally be computed by superimposing the flow provided by the fluid solver and the velocity originating from the various vortices $i: \mathbf{v}=\mathbf{v}_{f}+\sum_{i=1}^{n} \mathbf{v}_{\text {vort } i}$.

\section{Results and Discussion}

The left image in Figure 1 illustrates a surface mesh of a uterus. The vertices are labeled according to their behavior during the subsequent deformations. The white vertices, highlighted with the ellipse, represent the portion of the surface protruding into the vagina which is fixed prior to surgery. The blue vertices (inner surface) represent the endometrium deforming according to the pressure induced by the fluid. The surrounding organs like the urinary bladder and the rectum can be considered as being quite soft compared to the uterus. Therefore, the remaining vertices colored in red (outer surface) are free to move. The uterus in the middle corresponds to the surface of the tetrahedral mesh consisting of

Table 1. Performance data

\begin{tabular}{lcccccccc}
\hline \multicolumn{2}{l}{\begin{tabular}{l}
\multicolumn{6}{l}{ voxel } \\
{$[\mathrm{cm}]$}
\end{tabular}} & \#voxels & \#voxels CPU time $[\mathrm{ms}]$ & CPU time $[\mathrm{ms}]$ & Voxelization & Time to traverse Fidelity \\
& fluid & no vortices & with vortices & {$[\mathrm{ms}]$} & {$[\mathrm{s}]$} & \\
\hline 0.06 & 191022 & 35179 & 100 & 112 & 77 & 5 & + \\
0.09 & 62964 & 9528 & 26 & 30 & 42 & 2 & ++ \\
0.12 & 27880 & 3813 & 10 & 12 & 28 & 1.5 & ++ \\
0.15 & 15246 & 1818 & 5 & 6 & 19 & 1 & - \\
0.18 & 9408 & 969 & 3 & 5 & 12 & $<1$ & - \\
\hline
\end{tabular}




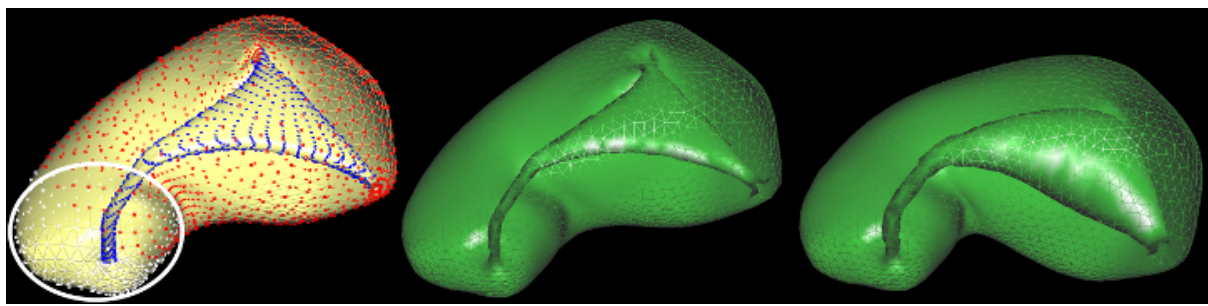

Fig. 1. Left: Uterus with labeled vertices. Middle: Corresponding surface of tetrahedra mesh. Right: uterus with hydrometra. In all cases the frontal surface has been rendered in wireframe mode to visualize the inner cavity.

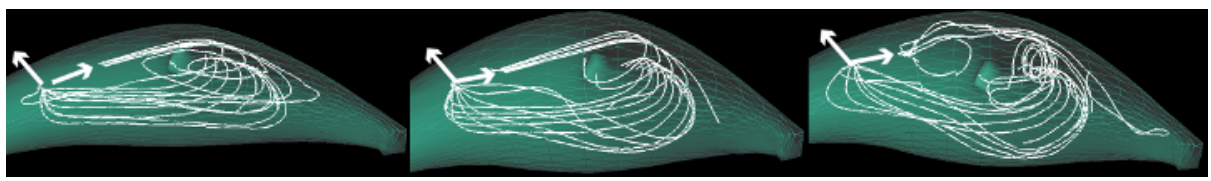

Fig. 2. Left: Visualization of the flow in the uterine cavity under limited pressure. Middle: Flow pathlines in a fully distended state. Right: Effect of additional vortices on the flow field.

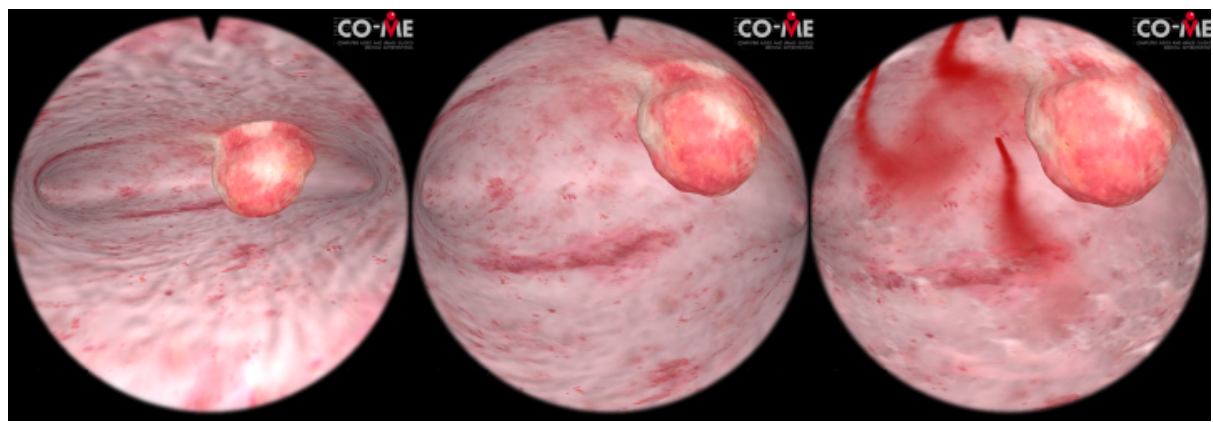

Fig. 3. Left: Low hydrometra in a cavity with a myoma. Middle: Same scene under maximal distension. Right: Scene with blood streams under the influence of both inflow and vortices.

approximately 35'000 tetrahedra. Finally, the uterus on the right illustrates the result of the FEM-based hydrometra simulation as presented.

During surgery the interaction is mainly restricted to the fundus of the uterus. Therefore, the simulation is confined to this region of interest. In Figure 2 the flow field is visualized by pathlines. Two different stages of hydrometra are shown in the first two images, while the third image illustrates the flow in the presence of 20 vortices. The arrows indicate the inflow and outflow directions. As can be seen, the pathlines clearly indicate the main flow streams from the inlet position to the outflow. The vortices clearly perturb the local behavior as desired, but do not disrupt the overall flow. 
Table 1 compiles different performance measurements while using the presented algorithms on a standard $3.0 \mathrm{GHz}$ PC. As the computational time for the deformation of the tetrahedral mesh can be neglected, the single parameter that determines the overall performance is the size of the voxels used for discretization. The table shows the values measured for increasing voxel dimensions. The total number of voxels in the bounding box of the organ are shown in the second column. The effective number of voxels used for flow simulation in the fully distended case are shown in the third column. Obviously, all calculations are faster for smaller hydrometra states. The next two columns indicate the CPU time for the flow field evaluation without and with up to 100 vortices. For every new state, the voxel representation has to be updated (6th column). While all these values prove that the update of the flow can be computed in real-time, the maximal flow speed that can be represented is limited by the time required to propagate a single particle across the cavity, indicated by the 'time to traverse'. The highest resolution is not optimal, as the numerical dissipation of the semi-Lagrangian method can be observed. Large voxel dimensions allow for the representation of faster flows, however, they are no longer able to accurately guide the particles from the in- to the outlet. In conclusion, an optimal voxel size of approximately $0.12 \mathrm{~cm}$ has been identified.

In order to validate the visual fidelity of the flow field we use blood streams as they are highly influenced by the distension liquid in real surgery. The blood streams are mostly computed on the GPU by using billboards and dynamic textures to represent the blood particles. Figure 3 shows three different snapshots of the simulator with a myoma attached to the upper wall. The dynamic behavior of the discussed images can be observed in the movie provided online (www.vision.ee.ethz.ch/ rsierra/hydrometra.avi).

As stated, the computation of fluid dynamics on the GPU is nowadays a field of intensive research. However, this approach is only of limited relevance in the current environment, as the information of the fluid field needs to be propagated to several other components of the simulator, such as the rendering of the blood streams, the displacement of floating tissue fragments or air bubbles. This requires copying of the results from the GPU to the CPU memory, an expensive operation in currently available hardware setups.

\section{Conclusion and Future Work}

In this paper we have presented the methods developed for the full simulation of hydrometra during hysteroscopy. Both the organ's deformation and the fluid flow in the cavity are modeled with highest possible realism while still meeting the real-time requirements of a simulator.

The fluid simulation will be further validated. We plan to perform an accurate flow simulation with exactly the same boundary conditions with an available commercial packages offline and quantitatively compare to our own results. The increased computational power of future hardware can be used to enhance the accuracy of the flow simulation. The Lattice Boltzmann Method 16] offers an 
appealing alternative to account for additional aspects of the underlying physics and will be implemented in the future.

The presented FEM computation is simplified in several respects. Many assumptions, e.g. quasi static behavior, may not hold for a realistic simulation. It might be necessary to resort to more complex models incorporating non-linear, anisotropic, and time dependent materials which approximate the real behavior of the myometrium more precisely. Therefore, a study is currently being performed where the hydrometra is generated in a surgically removed uterus in a CT scanner. This study will provide the necessary ground truth for the derivation of more advanced FEM models in the future.

Acknowledgment. This work has been supported by the NCCR CO-ME of the Swiss National Science Foundation. We are grateful to Stephan Weiss for his help with the FEM models and the use of MarcMentat ${ }^{\mathrm{TM}}$.

\section{References}

1. J. D. Anderson et al. Computational Fluid Dynamics: The Basics with Applications. McGraw-Hill, Inc., New York, 1995.

2. ACOG Tech. Bulletin. Hysteroscopy. Int J Gyn. Obstet., May 1994. 45(2): 175-180.

3. N. Foster and D. Metaxas. Realistic animation of liquids. Graphical Models and Image Processing, 58(5):471-483, 1996.

4. E. Gladilin et al. A biomechanical model for soft tissue simulation in craniofacial surgery. In Medical Imaging and Augmented Reality, pages 137-141, 2001.

5. M. Jiang, R. Machiraju, and D. Thompson. Geometric verification of swirling features in flow fields. In Visualization '02, pages 307-314, 2002.

6. Y. Liu, X. Liu, and E. Wu. Real-time 3D fluid simulation on GPU with complex obstacles. In Pacific Graphics, pages 247-256, 2004.

7. L. Mencaglia and E. Hamou. Manual of gynecological hysteroscopy - diagnosis and surgery. Endo-Press, Germany, 2001.

8. A. Nava et al. Evaluation of the mechanical properties of human liver and kidney through aspiration experiments. Technology and Health Care, 12(3):269-280, 2004.

9. J.C. Petrozza. Hysteroscopy. http://www.emedicine.com/med/topic3314.html, July 2004.

10. G. Picinbono et al. Non-linear and anisotropic elastic soft tissue models for medical simulation. In Intl. Conf. on Robotics and Automation, pages 1370-1375, 2001.

11. T. Sederberg and S. Parry. Free-form deformation of solid geometric models. Computer Graphics, 4:151-160, 1986.

12. R. Sierra et al. Coherent scene generation for surgical simulators. In Proceedings of ISMS 2004, pages 221 - 229. Springer Verlag, June 2004.

13. R. Sierra et al. Generation of variable anatomical models for surgical training simulators. Medical Image Analysis, to appear.

14. J. Stam. Stable fluids. In Proceedings of the 26th annual conference on Computer graphics and interactive techniques, pages 121-128, 1999.

15. D. Terzopoulus and K. Waters. Analysis and synthesis of facial image sequences using physical and anatomical models. IEEE Trans. PAMI, 15(6):569-579, 1993.

16. X. Wei et al. Blowing in the wind. In Proceedings of the 2003 ACM SIGGRAPH/Eurographics Symposium, pages 75-85, 2003.

17. S. Weiss et al. A finite element model for the simulation of hydrometra. Technology and Health Care, 12:259-267, 2004.

18. J. Zátonyi et al. Real-time synthesis of bleeding for virtual hysteroscopy. Medical Image Analysis, 9(3):255-266, June 2005. 